\title{
RAKE RECEPTION FOR UWB COMMUNICATION SYSTEMS WITH INTERSYMBOL INTERFERENCE
}

\author{
A.G. Klein ${ }^{\dagger}$, D.R. Brown III ${ }^{\ddagger}$, D.L. Goeckel ${ }^{\S}$, and C.R. Johnson, Jr. ${ }^{\dagger}$ \\ $\dagger$ Cornell University, School of Electrical and Computer Engineering, Ithaca, NY 14853 \\ $\ddagger$ Worcester Polytechnic Inst., Dept. of Electrical and Computer Engineering, Worcester, MA 01609 \\ $\S$ University of Mass., Dept. of Electrical and Computer Engineering, Amherst, MA 01003
}

\begin{abstract}
Recently, ultra wideband (UWB) technology has been proposed for use in wireless personal area networks (WPANs). Under the conditions where such transceivers are expected to operate, intersymbol interference (ISI) will become a significant performance limitation, and improvements to conventional RAKE reception will be necessary. We propose a modified RAKE receiver that finds an optimal balance between the goal of gathering multipath signal energy, avoiding ISI, and suppressing narrowband interference. For fixed RAKE finger delays, we develop a closed-form expression for the minimum mean squared error (MMSE) combining weights that account for ISI. We then examine the optimal choice of RAKE finger delays, and show that significant performance gains can be achieved, particularly in an undermodeled situation when there are more channel paths than RAKE fingers. Several numerical examples are presented which compare our proposed scheme to a conventional RAKE with maximal ratio combining (MRC).
\end{abstract}

\section{INTRODUCTION}

Ultra wideband (UWB) communication has attracted recent interest in the research and standardization communities, due to its promising ability to provide high data rate at low cost and power consumption. Another frequently cited benefit of UWB transmission is its ability to resolve individual multipath components (see, for instance, [1]). This feature motivates the use of RAKE multipath combining techniques to provide diversity and capture as much energy as possible at the UWB receiver.

UWB is being considered for use in wireless personal area networks (WPANs), where required data rates are in excess of $110 \mathrm{Mbps}$ per user. To obtain such rates with binary signaling, the required symbol period will need to be less than $10 \mathrm{~ns}$ even without coding or spreading. Meanwhile, several UWB indoor channel measurement campaigns

\footnotetext{
${ }^{\dagger}$ Supported in part by Texas Instruments and a Lockheed Martin Fellowship.

${ }^{\S}$ Supported in part by a MURI Project under Contract DAAD10-01-1-0477.
}

have demonstrated delay spreads far beyond $30 \mathrm{~ns}$ [2][3], which indicate that significant intersymbol interference (ISI) is unavoidable. A RAKE receiver, the preferred structure for collecting multipath energy in UWB systems, does not combat ISI. The vast majority of published results on UWB have overlooked this fact as most performance analyses employ a RAKE receiver under the assumption that channel delay spreads are much less than the pulse repetition period [4]. In this paper, the RAKE structure is modified to one that considers ISI and narrowband interference. By minimizing the mean squared error (MSE), we arrive at a choice of RAKE delays and combining weights that yield superior performance when compared to maximal ratio combining (MRC). A generalized RAKE for combatting interference was previously proposed in the context of DS-CDMA [5], where the authors require the noise to be white Gaussian, and they employ a different optimization criterion (i.e. maximum-likelihood) but arrive at a similar result. A modified RAKE for suppressing narrowband interference was proposed in [6], but no consideration is given to ISI.

\section{SYSTEM MODEL}

Two popular modulation types being considered for UWB are pulse-position modulation (PPM) and pulse amplitude modulation (PAM). Here, we consider systems of the PAM type, and a model for single-user M-PAM UWB transmission over channels with ISI is shown in Figure 1. We have assumed a baseband transmission system, and hence all signals are assumed to be real-valued. The zero-mean i.i.d. data symbols $\left\{s_{n}\right\}$ are passed through a unit energy pulseshaping filter $p(t)$ which includes the effects of the transmit antenna. Note that we require the pulse shape to be unchanging from symbol period to symbol period, but our model is general enough to include either time hopping (TH) or direct sequence (DS) block spreading if, for example, $p(t)$ is the sum of several delayed Gaussian monocycles. After pulse shaping, the signal undergoes the effects of a 


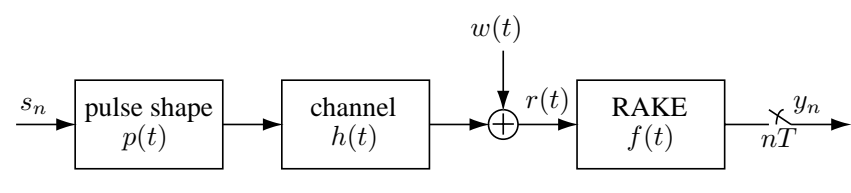

Fig. 1. System model.

channel with $\mathrm{L}$ paths whose response given by:

$$
h(t)=\sum_{\ell=0}^{L-1} \alpha_{\ell} \delta\left(t-\tau_{\ell}\right)
$$

where $\alpha_{\ell}$ and $\tau_{\ell}$ are the gain and delay introduced by the $\ell$ th path of the channel. The received signal can then be expressed as:

$$
r(t)=\sum_{i=-\infty}^{\infty} s_{i} \sum_{\ell=0}^{L-1} \alpha_{\ell} p\left(t-\tau_{\ell}-i T\right)+w(t)
$$

where $T$ is the symbol rate and $w(t)$ is additive noise. The noise is assumed to be a zero-mean wide sense stationary process that is uncorrelated with the data, and it may be colored due to narrowband interferers.

In the case of no ISI and when the noise is AWGN, the optimal receiver is a filter matched to the received waveform (i.e. the combined response of the channel and transmit pulse shapes). Typically, this is implemented in a RAKE receiver structure with $M$ fingers, which can be represented as a filter with response:

$$
f(t)=\sum_{m=0}^{M-1} \beta_{m} p\left(-t-\theta_{m}\right)
$$

where our model places no restrictions on the spacing of the RAKE delays $\theta_{m}$. The sampled output of the RAKE receiver is then:

$$
\begin{aligned}
y_{n} & =\left.[r(t) \star f(t)]\right|_{t=n T} \\
= & \sum_{i=-\infty}^{\infty} s_{i} \sum_{\ell=0}^{L-1} \sum_{m=0}^{M-1} \alpha_{\ell} \beta_{m} R_{p}\left(n T-i T+\theta_{m}-\tau_{\ell}\right) \\
& +\tilde{w}(n T)
\end{aligned}
$$

where $R_{p}(t) \triangleq \int_{-\infty}^{\infty} p(\tau) p(\tau+t) d \tau$ is the time-autocorrelation of the pulse shape and

$$
\tilde{w}(t)=\sum_{m=0}^{M-1} \beta_{m} \int_{-\infty}^{\infty} w(\tau) p\left(-t+\tau-\theta_{m}\right) d \tau
$$

is filtered noise.

It is well known that the optimal combiner for the AWGN multipath channel is MRC, where $M=L$ fingers, $\beta_{m}=$ $\alpha_{m}$, and $\theta_{m}=\tau_{m}$. When the received signals on each finger are orthogonal (as is the case when there is no ISI), MRC attains the matched filter bound [7]. However, when the ISI becomes significant the orthogonality of the paths is violated unless care is taken in the design of the pulse shape (as in long-coded DS-CDMA systems where successive symbols are nearly orthogonal). For UWB-based high rate WPANs it is anticipated that spreading codes will be short, and therefore MRC is suboptimal even when the noise is AWGN. This motivates a smarter choice of combining weights $\beta_{m}$ and finger delays $\theta_{m}$ to combine the signal energy while compensating for the effects of ISI and narrowband interference.

\section{ISI-AWARE RAKE}

In the presence of ISI, the optimal receiver is the maximumlikelihood sequence estimator (MLSE). Since the computational complexity grows exponentially with channel length, most channels of practical interest require too much computation for MLSE to be feasible. Consequently, system designers typically resort to suboptimal schemes like linear equalization to compensate for ISI. A linear equalizer and a RAKE are structurally quite similar, as both are linear combiners. However, an equalizer operates on sampled data, whereas a RAKE does not need to be confined to a "grid" of delays imposed by sampling. Furthermore, an equalizer attempts to suppress ISI by minimizing some metric like MSE, while a MRC-RAKE ignores ISI and attempts to gather all the signal energy to maximize the SNR. In this section, we propose a RAKE where the finger delays and combining weights are chosen to minimize the MSE, thereby arriving at a RAKE that finds an optimal balance between the goal of gathering multipath signal energy, avoiding ISI, and combatting narrowband interference. In the analysis that follows, we assume complete knowledge of the channel path delays and gains, the transmit pulse shape, and the autocorrelation of the additive noise.

\subsection{Choosing the Combining Weights}

Define $\mathbf{b}=\left[\begin{array}{llll}\beta_{0} & \beta_{1} & \ldots & \beta_{M-1}\end{array}\right]^{\top}$ to be the vector of RAKE combining weights, and define $\Phi[n] \in \mathbb{R}^{M}$ as:

$$
\Phi[n] \triangleq \sum_{\ell=0}^{L-1} \alpha_{\ell}\left[\begin{array}{c}
R_{p}\left(n T-\tau_{\ell}+\theta_{0}\right) \\
R_{p}\left(n T-\tau_{\ell}+\theta_{1}\right) \\
\vdots \\
R_{p}\left(n T-\tau_{\ell}+\theta_{M-1}\right)
\end{array}\right]
$$

so that $\mathbf{b}^{\top} \Phi[n]$ represents the combined response of the pulse shaping, channel, and RAKE, and (1) can be represented as:

$$
y_{n}=\sum_{i=-\infty}^{\infty} s_{i} \mathbf{b}^{\top} \Phi[n-i]+\tilde{w}(n T)
$$


Define the matrix $\mathbf{R} \in \mathbb{R}^{M \times M}$ as $[\mathbf{R}]_{i, j} \triangleq \int_{-\infty}^{\infty} R_{w}(\tau) R_{p}(\tau+$ $\left.\theta_{i}-\theta_{j}\right) d \tau$, where $R_{w}(\tau) \triangleq E[w(t) w(t+\tau)]$ is the statistical autocorrelation of the noise. Due to the assumption of a time-invariant channel, the MSE is given by:

$$
\begin{aligned}
J_{\Theta, \mathbf{b}}= & E\left[\left|s_{n}-y_{n}\right|^{2}\right] \\
= & \sigma_{s}^{2}\left[1-2 \mathbf{b}^{\top} \Phi[0]\right. \\
& \left.+\mathbf{b}^{\top}\left(\frac{1}{\sigma_{s}^{2}} \mathbf{R}+\sum_{i=-\infty}^{\infty} \Phi[i] \Phi^{\top}[i]\right) \mathbf{b}\right]
\end{aligned}
$$

where we have used the fact that the data symbols and sampled noise process are uncorrelated, and $\sigma_{s}^{2}=E\left[s_{n}^{2}\right]$ is the power of the source symbols.

We see from (2) that the MSE is quadratic in $\mathbf{b}$, and hence the globally optimal solution to minimize the MSE is given by:

$$
\mathbf{b}_{o p t}=\left(\frac{1}{\sigma_{s}^{2}} \mathbf{R}+\sum_{i=-\infty}^{\infty} \Phi[i] \Phi^{\top}[i]\right)^{-1} \Phi[0]
$$

So, with full knowledge of the channel, we have a formula for the optimal combining weights for a given set of RAKE finger delays. Note also that we do not require the number of channel paths $L$ to equal the number of RAKE fingers $M$, so (3) is valid in the undermodeled case when $M<L$. Furthermore, since the cost surface is quadratic, stochastic gradient descent techniques can be applied to adaptively determine $\mathbf{b}_{\text {opt }}$.

In the special case when the additive noise is white and Gaussian so that $R_{w}(\tau)=N_{0} \delta(\tau) / 2$, the combining weights that minimize the MSE given by (3) coincide with the combining weights that yield the maximum-likelihood detector given in [5]. In the special case when there is no ISI so that $\Phi[0]=\left[\begin{array}{lll}\alpha_{0} \ldots \alpha_{M-1} & \ldots\end{array}\right]^{\top}$ and $\Phi[n]=\mathbf{0}, \forall n \neq 0$, the combining weights that minimize the MSE given by (3) coincide with the combining weights given in [6].

\subsection{Choosing the Finger Delays}

The prior section showed that the optimal choice of combining weights $\beta_{m}$ is uniquely determined by the choice of finger delays $\theta_{m}$ in the RAKE receiver. In the typical case where the RAKE finger delays are matched to the channel path delays $\left(\theta_{m}=\tau_{m}\right)$ and the RAKE order is equal to the channel order $(L=M)$, it is clear that the optimal combining weights of the generalized RAKE receiver will achieve a performance gain with respect to the standard MRC weights in the presence of ISI. It is not as clear, however, if any gain can be achieved by allowing the generalized RAKE receiver to select finger delays different from the channel path delays. This section considers the problem of finding optimal finger delays for the generalized RAKE receiver. Specifically, in the case when $\beta_{m}$ is selected optimally (3), the MSE cost can be expressed strictly as a function of the finger delays:

$J_{\Theta}=\sigma_{s}^{2}\left[1-\Phi[0]^{\top}\left(\frac{1}{\sigma_{s}^{2}} \mathbf{R}+\sum_{i=-\infty}^{\infty} \Phi[i] \Phi^{\top}[i]\right)^{-1} \Phi[0]\right]$

and the optimal RAKE finger delays can be found via:

$$
\begin{aligned}
\Theta_{\text {opt }}=\arg \max _{\Theta} & {\left[\Phi [ 0 ] ^ { \top } \left(\frac{1}{\sigma_{s}^{2}} \mathbf{R}\right.\right.} \\
& \left.\left.+\sum_{i=-\infty}^{\infty} \Phi[i] \Phi^{\top}[i]\right)^{-1} \Phi[0]\right]
\end{aligned}
$$

where $\Theta=\left[\begin{array}{llll}\theta_{0} & \theta_{1} & \ldots & \theta_{M-1}\end{array}\right]^{\top}$ is the vector of RAKE finger delays. Unfortunately, this approach does not lead to a manageable closed-form expression for $\Theta$, and the resulting function has many local extrema as will be shown in the following numerical examples. Nevertheless, the examples will also demonstrate that choosing the RAKE delays as in (4) often leads to significantly lower MSE than by choosing the delays as in MRC.

\section{NUMERICAL RESULTS}

In this section we plot the cost surface for the case when there are just two RAKE fingers, thereby permitting visualization of the cost surface and its local extrema. The pulse shape is chosen to be the familiar unit energy Gaussian monocycle given by:

$$
p(t)=\sqrt{\frac{8}{3 \tau^{5}}}\left(\tau^{2}-4 \pi t^{2}\right) \exp \left(-2 \pi(t / \tau)^{2}\right)
$$

where the parameter $\tau=250 \mathrm{ps}$ corresponds to a pulse width of about 500 ps. For all examples, the pulse repetition period $T=2 \mathrm{~ns}$, and the noise is assumed to be AWGN. In the following discussion, $\mathbf{b}_{\text {opt }}$ and $\Theta_{o p t}$ refer to the choice of parameters as in (3) and (4), whereas $\mathbf{b}_{m r c}$ and $\Theta_{m r c}$ refer to the choice of parameters as in MRC. The cost surfaces are plotted in the $\Theta$-space for $\mathbf{b}$ chosen as in (3); the stars indicate $\Theta_{o p t}$, while the triangles indicate $\Theta_{m r c}$.

\subsection{Short channel: $M=L=2$}

Consider the channel given by $L=2, \tau_{0}=0 \mathrm{~ns}, \tau_{1}=1.96$ ns, $\alpha_{0}=0.89$, and $\alpha_{1}=-0.44$. The MSE cost surface is shown in Figure 2. In the noise-dominated regime $\left(\mathrm{SNR}=0 \mathrm{~dB}\right.$ ), we see that $\Theta_{o p t}$ and $\Theta_{m r c}$ coincide (as do $\mathbf{b}_{\text {opt }}$ and $\left.\mathbf{b}_{m r c}\right)$, and yield the same MSE; both schemes 

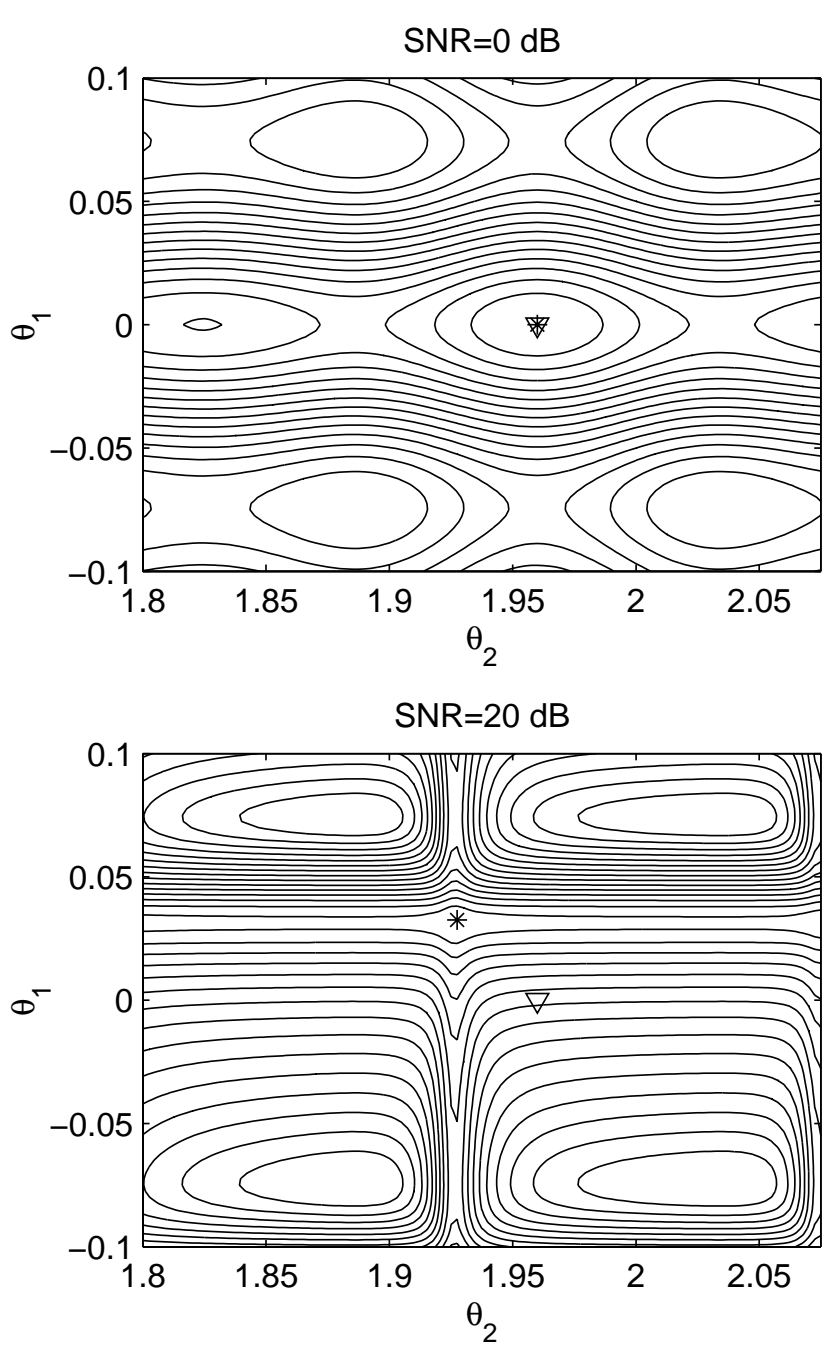

Fig. 2. MSE cost surface for short channel case $\left(\nabla=\Theta_{m r c}\right.$, $\left.*=\Theta_{\text {opt }}\right)$.

attempt to capture as much energy as possible. In the ISIdominated regime $(\mathrm{SNR}=20 \mathrm{~dB})$, the minimum of the surface has moved to a new location, and now the first RAKE finger should be more heavily weighted. For the high SNR example, the MMSE parameters yield a MSE that is $8.4 \mathrm{~dB}$ better than the MRC parameters. At low SNR, the optimal RAKE approximates a matched filter, while at high SNR (where noise amplification is not an issue) it approximates a channel inverse filter. The graph of the cost surface in the $\Theta$-space shows evidence of local minima.

\subsection{Undermodeled Case: $M=2, L=14$}

Now consider the channel with many clustered arrivals given by $L=14$, and $\tau=[0,0.01,0.012,0.02,0.34,0.57,0.77$, $0.82,1.21,1.37,1.52,1.81,1.88,1.96]$ and $\alpha=[0.139,0.695$,

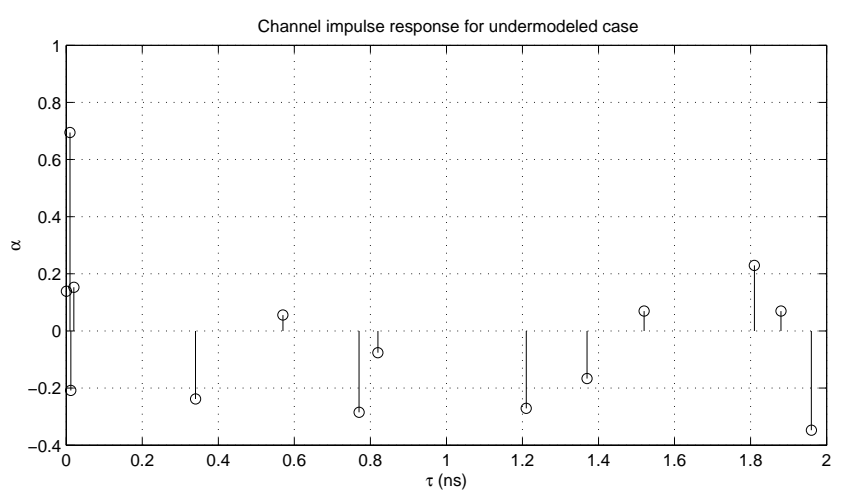

Fig. 3. Channel impulse response for the undermodeled case.

$-0.209,0.153,-0.238,0.056,-0.285,-0.076,-0.271,-0.167$, $0.066,0.229,0.07,-0.347]$ which has impulse response shown in Figure 3. Keeping the number of RAKE fingers at $M=$ 2 , the MSE cost surface is shown in Figure 4 for an ISIdominated regime of $20 \mathrm{~dB}$ SNR. For this example, the 2finger RAKE with MMSE parameters yields a MSE that is $4.6 \mathrm{~dB}$ better than the MRC parameters with the full $M=$ 14 fingers. Once the SNR falls below $13.1 \mathrm{~dB}$, the MMSE and MRC solutions achieve the same MSE. Note that the MRC solution is not identified in Figure 4 since it has 14 fingers. This result further illustrates the importance of making smart choices of the RAKE parameters in the presence of ISI, and shows that fewer RAKE fingers are required when the delays and combining weights are chosen appropriately.

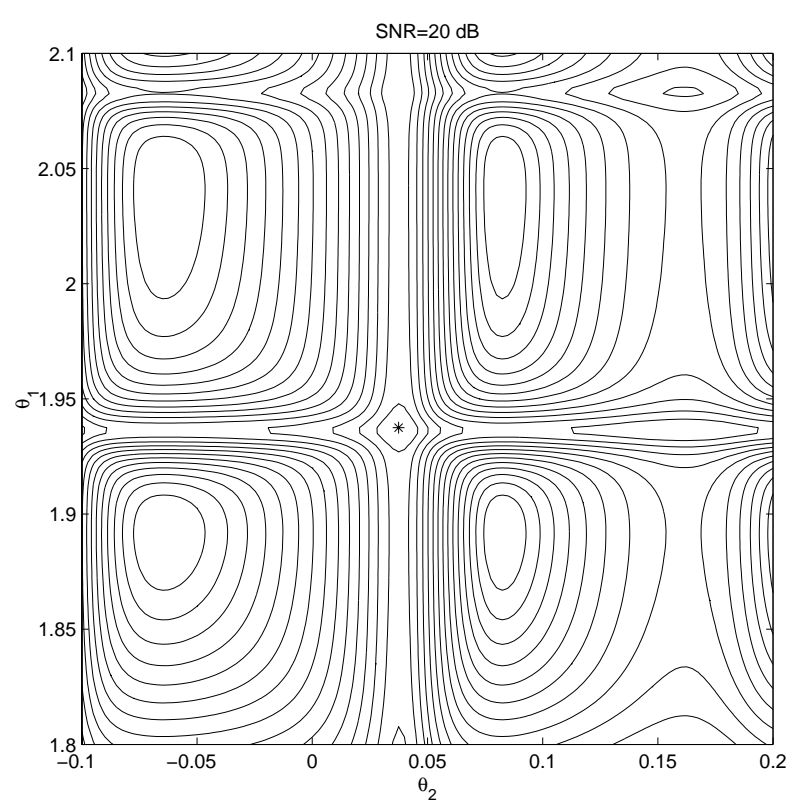

Fig. 4. MSE cost surface for long channel. 


\section{CONCLUSION}

For high data rate UWB systems, orthogonality between each of the signals in the various RAKE fingers is an invalid assumption, and this oversight may lead to significant performance degradation. A modified RAKE receiver has been proposed that finds an optimal balance between the goal of gathering multipath signal energy, avoiding ISI, and combatting narrowband interference. The proposed receiver demonstrates superior performance over conventional MRC when the ISI dominates the noise. For fixed RAKE finger delays, we showed how to find the MMSE combining weights that account for ISI; finding these weights is not difficult, and could be done adaptively. We then examined an optimal choice of RAKE finger delays, and showed that even further performance gains can be achieved, especially in the undermodeled case when the number of RAKE fingers is less than the number of channel paths. Nevertheless, there are some difficulties with finding the optimal finger delays due to the non-quadratic nature of the cost surface and the presence of local minima with large regions of attraction in the finger delay-space. Efficient solutions to the optimal finger delay problem remain an open problem.

\section{ACKNOWLEDGEMENTS}

The authors would like to thank Professor Joon Ho Cho of University of Massachusetts Amherst for fruitful discussions.

\section{REFERENCES}

[1] M.Z. Win and R.A. Scholtz, "Ultra-Wide Bandwidth Time-Hopping Spread-Spectrum Impulse Radio for Wireless Multiple-Access communications," Transactions on Communications, vol. 48, pp. 679-689, April 2000.

[2] R.A. Scholtz and M.Z. Win, "On the Robustness of Ultra-Wide Bandwidth Signals in Dense Multipath Environments," Communications Letters, vol. 2, pp. 5153, Feb 1998.

[3] J. Keignart and N. Daniele, "Subnanosecond UWB Channel Sounding in Frequency and Temporal Domain," Proc. 2002 IEEE Conf on UWB Systems and Technologies, May 2002.

[4] J.D. Choi and W.E. Stark, "Performance of UltraWideband Communications with Suboptimal Receivers in Multipath Channels," Journal on Selected Areas in Communications, vol. 20, pp. 1754-1766, Dec. 2002.
[5] G.E. Bottomley, T. Ottosson, and Y.E. Wang, "A Generalized RAKE Receiver for Interference Suppression," Journal on Selected Areas in Communications, vol. 18, pp. 1536-1545, Aug 2000.

[6] I. Bergel, E. Fishler, and H. Messer, "Narrow-band Interference Suppression in Time-Hopping ImpulseRadio Systems," Proc. 2002 IEEE Conf on UWB Systems and Technologies, May 2002.

[7] J.G. Proakis, Digital Communications, 4th ed, McGraw-Hill Book Company, New York, 2000. 